\title{
Study on Vapor-Liquid Nucleation Rates for $n$-Alcohols by Density Functional Theory
}

\author{
Wu, Huijie(吴慧杰)Ｌi, Xiaosen*(李小森) \\ Key Laboratory of Renewable Energy and Gas Hydrate, Guangzhou Institute of Energy Conversion, Chinese \\ Academy of Sciences, Guangzhou, Guangdong 510640, China
}

\begin{abstract}
The statistical associating fluid theory (SAFT) in conjunction with the Weeks-Chandler-Anderson (WCA) approximation for intermolecular interaction is employed to construct a non-uniform equation of state (EOS) for $n$-alcohols. The molecular parameters for methanol, ethanol, 1-propanol, 1-butanol, 1-pentanol and 1-hexanol are obtained by fitting to the experimental data of vapor-liquid equilibria and then used to predict the nucleation rates under the framework of density functional theory (DFT). The predictions are found to be in quite good agreement with the experimental data. Investigation shows that the combination of DFT and SAFT is a successful approach for vapor-liquid nucleation rates of $n$-alcohols.
\end{abstract}

Keywords SAFT, WCA, DFT, vapor-liquid equilibria, nucleation rates

\section{Introduction}

Nucleation refers to the kinetic processes involved in the initiation of first order phase transitions in non-equilibrium systems. Vapor-liquid nucleation of dipolar and associating fluids is of fundamental and practical importance in atmospheric studies, and much effort has been devoted in the recent years to understanding the properties of critical nuclei in such types of systems.

The first comprehensive treatment of the thermodynamics of the nucleation process is due to Gibbs, ${ }^{1}$ who showed the reversible work required to form a nucleus of the new phase consists of a bulk term and a surface term, which can be evaluated by using the bulk thermodynamic properties. Years later, this theory was developed by Volmer and Weber, ${ }^{2}$ Farkas, ${ }^{3}$ Becker and doring, ${ }^{4}$ Frenkel ${ }^{5}$ and Zeldovich, ${ }^{6}$ and now is known as classical nucleation theory (CNT) ${ }^{7-10}$ CNT has been successful in the prediction of critical supersaturations in simple non-polar fluids. ${ }^{11,12}$ However, it tends to predict too high nucleation rates at high temperatures and too low rates at the lower temperatures due to inappropriate treatment of curvature contributions to the work of formation of the critical nuclei. Moreover, this approach unsatisfactorily estimates the critical supersaturations of associating fluids such as water, methanol and other short-chain $n$-alcohols. ${ }^{13-15}$

Due to the limits of CNT, there has been much effort to improve the classical model, e.g., the Scale model ${ }^{16,17}$ and Katz-Saltsburg-Reiss (KSR) model ${ }^{18}$ have been proposed to describe the vapor-liquid nucleation for associating fluids. The Scale model is based on CNT and yields a nearly universal dependence of nucleation rate on $T_{\mathrm{c}} / T-1$. By introducing an excess surface entropy parameter $(\approx 1.5$ for dipolar fluids) and a constant rate prefactor $\left(\approx 10^{26} \mathrm{~cm}^{-3} \cdot \mathrm{s}^{-1}\right)$, the Scale model is successful for dipolar fluids including water and heavy water. The KSR model is also based on CNT but the effect of association between vapor particles on the rate of nucleation is taken into account. Although this model still overestimates the values of the critical supersaturations for most associating fluids, its predictions are in better agreement with the experimental data than those from CNT. Additionally, its predictions for the work of formation and number of particles in the critical nuclei are more reasonable. ${ }^{19}$ However, the modified models based on CNT are often substance specific and dependent on the bulk thermodynamic properties, which limits their applicability.

To avoid the substance specific characteristic and the simplistic use of bulk thermodynamic properties in evaluating the free energy of cluster formation, DFT ${ }^{20-22}$ is the most useful approach. Cahn and Hilliard ${ }^{23,24}$ are the first who developed a type of DFT-square gradient theory for nucleation theory. A more general form of DFT was developed by Oxtoby and coworkers. ${ }^{25-28}$ Compared with the classical approaches like CNT and KSR model, DFT allows for a more realistic treatment of curvature effects on the free energy of formation of critical clusters and predicts a vanishing barrier to nu-

* E-mail: lixs@ms.giec.ac.cn

Received March 3, 2010; revised and accepted June 25, 2010.

Project supported by the National Natural Science Foundation of China (No. 20773133), Science \& Technology Program of Guangdong Province (No. 2009B050600006), CAS Knowledge Innovation Program (No. KGCX2-YW-3X6) and CAS Magnitude Science and Technology Apparatus Development Program (No. YZ200717). 
cleation at the thermodynamic spinodal. Moreover, it can be used to calculate bulk and interfacial properties of the systems of interest.

By far, most of the DFT studies on vapor liquid nucleation have focused on non-polar fluids. ${ }^{25-40}$ Talanquer and Oxtoby ${ }^{19}$ extended DFT to the study of nucleation of associating fluids with a single association site. Talanquer $^{40}$ used DFT of statistical mechanics in a square gradient approximation to analyze the structure, size, and work of formation of critical nuclei in self-associating fluids. $\mathrm{Fu}$ and $\mathrm{Li}^{41}$ used DFT to study the nucleation of water and heavy water. However, the mentioned DFT work was limited to the cases of model like or spherical associating particles instead of chain like $n$-alcohols. Recently, $\mathrm{Fu}$ and Liu ${ }^{42}$ used a density gradient theory (DGT) to correlate the nucleation rates for $n$-alcohols ( $n$ from 1 to 6 ) and their results showed that DGT preserves all the advantages of DFT in capturing the structure and properties of nucleus but gives much more accurate nucleation rates by adjusting the influence parameter. However, in the work of $\mathrm{Fu}$ and $\mathrm{Liu}^{42}$ the influence parameters were regressed by fitting to the experimental data of nucleation rates, hence their model has a lack of predictive function.

In this work, the Weeks-Chandler-Anderson (WCA) approximation $^{43}$ for intermolecular interaction and the statistical associating fluid theory (SAFT) ${ }^{44,45}$ are combined to construct a non uniform equation of state for chain like associating fluids. The molecular parameters of $n$-alcohols are obtained by fitting to the experimental data of vapor-liquid equilibria and then used to predict the nucleation properties under the framework of DFT.

\section{Theory}

The grand potential $\Omega[\rho(\boldsymbol{r})]$ of a vapor-liquid surface can be expressed as:

$$
\Omega[\rho(\boldsymbol{r})]=A[\rho(\boldsymbol{r})]-\mu \int \rho(\boldsymbol{r}) \mathrm{d} \boldsymbol{r}
$$

where $\rho(\boldsymbol{r})$ is the number density of molecules and $\mu$ is the chemical potential. According to SAFT, ${ }^{44,45}$ the local Helmholtz free energy functional, $A[\rho(\boldsymbol{r})]$, can be expressed as:

$$
\begin{aligned}
& A[\rho(\boldsymbol{r})]=A^{\mathrm{id}}[\rho(\boldsymbol{r})]+A^{\mathrm{hs}}[\rho(\boldsymbol{r})]+A^{\text {chain }}[\rho(\boldsymbol{r})]+ \\
& A^{\text {att }}[\rho(\boldsymbol{r})]+A^{\text {ass }}[\rho(\boldsymbol{r})]
\end{aligned}
$$

where $A^{\mathrm{id}}[\rho(\boldsymbol{r})], A^{\mathrm{hs}}[\rho(\boldsymbol{r})], A^{\text {chain }}[\rho(\boldsymbol{r})], A^{\mathrm{att}}[\rho(\boldsymbol{r})]$ and $A^{\text {ass }}[\rho(\boldsymbol{r})]$ correspond to the ideal gas, hard-sphere repulsion, chain formation, attractive interaction, and association contributions to the free energy of the system, respectively. SAFT is used here to formulate the Helmholtz free energy because it is based on extensions and simplifications of Wertheim's perturbation theory ${ }^{46,47}$ for associating fluids and has been widely used to model the vapor-liquid equilibria. Characterizing the chain like associating molecule by the diameter of a segment, the number of segments in the chain, the segment-segment dispersion energy, the association energy related to the change in enthalpy of association and the bond volume related to the change in entropy on association, SAFT is correlative and predictive for the phase behavior of most of the chain like associating fluids.

The ideal gas contribution in Eq. (2) is known exactly:

$$
A^{\mathrm{id}}[\rho(\boldsymbol{r})]=N k T \ln \left[\rho(\boldsymbol{r}) \Lambda^{3}-1\right]
$$

where $k$ is the Boltzmann constant, $T$ is the absolute temperature, and $\Lambda$ is the de Broglie thermal wavelength.

The hard-sphere repulsion is formulated in Carnahan-Starling equation: ${ }^{48}$

$$
A^{\mathrm{hs}}[\rho(\boldsymbol{r})]=N k T m \frac{4 \eta[\rho(\boldsymbol{r})]-3 \eta[\rho(\boldsymbol{r})]^{2}}{\{1-\eta[\rho(\boldsymbol{r})]\}^{2}}
$$

where $m$ is the number of segment in each molecule, $\eta[\rho(\boldsymbol{r})]=(\pi / 6) m \rho(\boldsymbol{r}) d^{3}$ is the packing factor and $d$ is an effective hard sphere diameter for segment: ${ }^{49,50}$

$$
\frac{d}{\sigma}=\frac{0.3837+1.068 / T^{*}}{0.4293+1 / T^{*}}
$$

where $\sigma$ and $\varepsilon$ are respectively the diameter and dispersion energy parameter for each segment, $T^{*}=k T / \varepsilon$ is the reduced temperature.

The association contribution is expressed as: ${ }^{44,45}$

$$
A^{\text {ass }}[\rho(\boldsymbol{r})]=N k T\left[M_{A} \ln \chi_{A}(\boldsymbol{r})-M_{A} \chi_{A}(\boldsymbol{r}) / 2+M_{A} / 2\right]
$$

where $M_{A}$ is the number of association sites on each molecule. According to the previous work, ${ }^{44,45} M_{A}=2$ is appropriate for $n$-alcohols. $\chi_{A}(\boldsymbol{r})$ is the fraction of molecules not bonded at site $A$, which is given by:

$$
\chi_{A}(\boldsymbol{r})=\frac{1}{1+\sum_{B} \rho(\boldsymbol{r}) \chi_{B}(\boldsymbol{r}) \Delta(\boldsymbol{r})}
$$

where $\Delta(\boldsymbol{r})=g^{\mathrm{hs}}\left[\exp \left(\varepsilon^{a} / k T\right)-1\right]\left(d^{3} \kappa^{a}\right)$ is related to the association strength, $\varepsilon^{a}$ is the association energy, and $d^{3} \kappa^{a}$ is a measure of the volume available for bonding of any two sites on different molecules. The radial distribution function for the hard-sphere fluid, $g^{\text {hs }}$, is given by:

$$
g^{\mathrm{hs}}=\{1-0.5 \eta[\rho(\boldsymbol{r})]\} /\{1-\eta[\rho(\boldsymbol{r})]\}^{3}
$$

The chain formation contribution is expressed as: ${ }^{44,45}$

$$
A^{\text {chain }}[\rho(\boldsymbol{r})]=N k T(1-m) \ln \left(g^{\mathrm{hs}}\right)
$$

The Helmholtz free energy functional due to the attractive interaction is expressed in a mean-field approximation: 


$$
A^{\text {att }}[\rho(\boldsymbol{r})]=\frac{1}{2 k T} \iint \mathrm{d} \boldsymbol{r}^{\prime} \mathrm{d} \boldsymbol{r} u^{\mathrm{WCA}}\left(\left|\boldsymbol{r}-\boldsymbol{r}^{\prime}\right|\right) m \rho(\boldsymbol{r}) m \rho\left(\boldsymbol{r}^{\prime}\right)
$$

where $m \rho(\boldsymbol{r})$ is the number density of segments at position $\boldsymbol{r}$. The Lennard-Jones potential in a WCA approximation is expressed as: ${ }^{43}$

$$
u^{\mathrm{WCA}}\left(\mid \boldsymbol{r}-\boldsymbol{r}^{\prime}\right)= \begin{cases}-\varepsilon, & \left|\boldsymbol{r}-\boldsymbol{r}^{\prime}\right| \leqslant r_{\mathrm{min}} \\ 4 \varepsilon\left(\frac{\sigma^{12}}{\left|\boldsymbol{r}-\boldsymbol{r}^{\prime}\right|^{12}}-\frac{\sigma^{6}}{\left|\boldsymbol{r}-\boldsymbol{r}^{\prime}\right|^{6}}\right), & \left|\boldsymbol{r}-\boldsymbol{r}^{\prime}\right|>r_{\min }\end{cases}
$$

$r_{\min }=\sqrt[6]{2} \sigma$

\section{Results and discussion}

\section{Bulk phases}

In the equilibrium bulk phases, the vapor density $\rho^{\mathrm{v}}$, liquid densities $\rho^{1}$, chemical potential $\mu$ and pressure $p^{\text {coex }}$ at given temperature can be determined according to the requirement that pressure and chemical potential in both phases should be equal. To accurately model the phase equilibria of $n$-alcohols, one needs five adjustable molecular parameters: number of segments in each molecule $m$, diameter of each segment $\sigma$, dispersion energy parameter of each segment $\varepsilon / k$, association volume of each association site $\kappa^{a}$ and association energy of each association site $\varepsilon^{a} / k$. These parameters can be regressed by fitting to the experimental data ${ }^{51,52}$ below the critical region. The optimized molecular parameters and the corresponding average relative deviations for saturation pressure $(p / \%)$ and liquid density $\left(\rho^{1} / \%\right)$ are shown in Table 1. The agreement between the calculated and experimental data of methanol is shown in Figure 1(a).

\section{Vapor-liquid nuclei}

Results of the equilibrium bulk properties can be used to derive the information about the structure and properties of the interface between coexisting phases. In particular, equilibrium density profiles can be determined by minimizing the grand potential $\Omega[\rho(\boldsymbol{r})]$. The condition $\delta \Omega[\rho(\boldsymbol{r})] / \delta \rho(\boldsymbol{r})=0$ leads to an Euler-Lagrange integral equation that can be solved via a standard Picard method with an initial guess of the density pro- file as primary input.
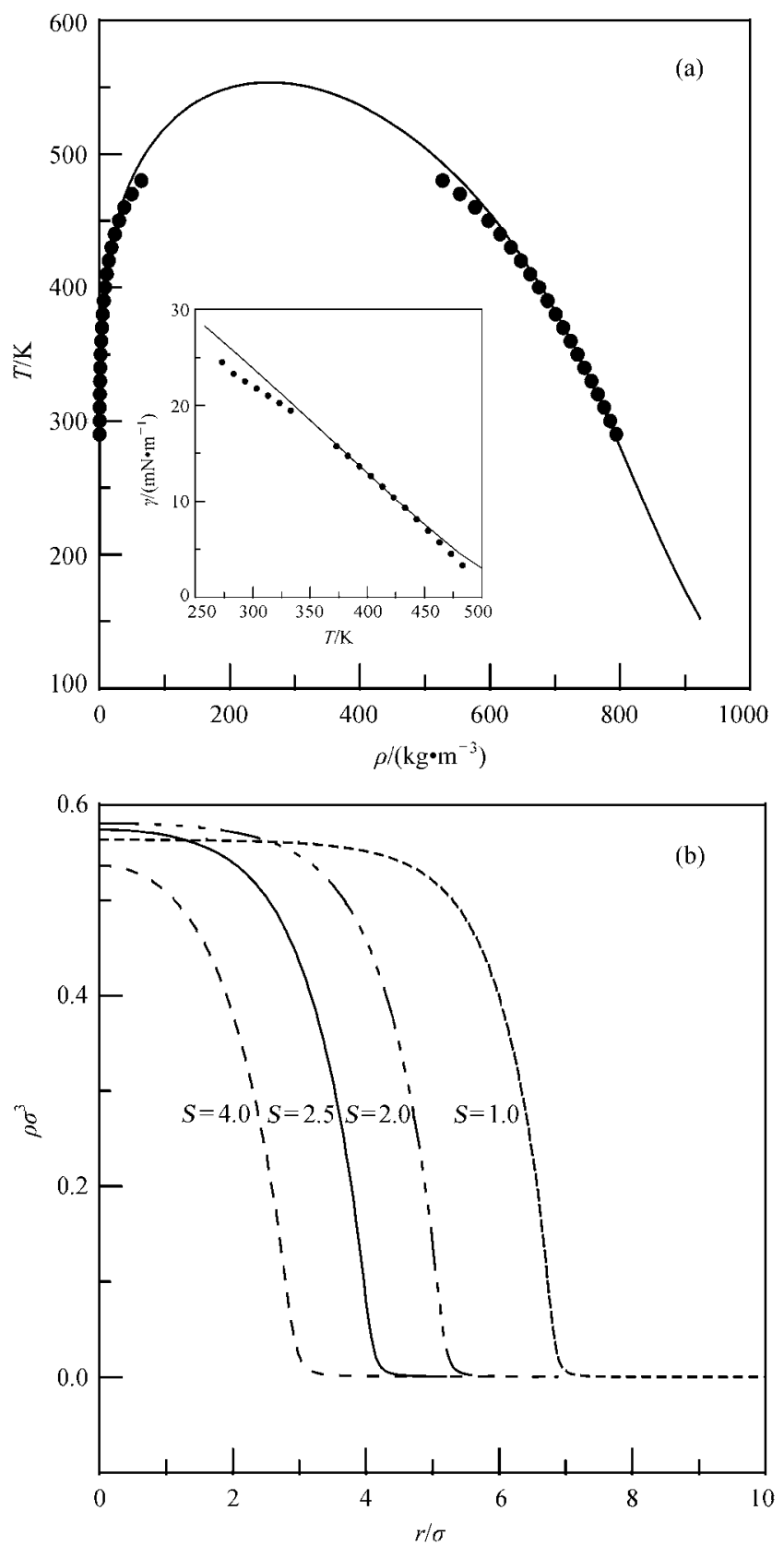

Figure 1 (a) Vapor-liquid equilibria and surface tensions (insert plot). Symbols: experimental data. ${ }^{51,52}$ (b) Equilibrium density profiles of vapor-liquid surface and vapor-liquid nuclei for methanol at given temperature $T=298.15 \mathrm{~K}$.

Table 1 Regressed parameters for chain like associating fluids ${ }^{a}$

\begin{tabular}{ccccccccc}
\hline & $m$ & $\sigma /\left(10^{-10} \mathrm{~m}\right)$ & $\varepsilon k^{-1} / \mathrm{K}$ & $\kappa^{a} \times 100$ & $\varepsilon^{a} k^{-1} / \mathrm{K}$ & $p / \%$ & $\rho^{1} / \%$ & $T$ range/K \\
\hline Methanol & 1.351 & 3.378 & 217.7 & 1.19 & 2889.6 & 1.3 & 1.4 & $290-480$ \\
Ethanol & 1.649 & 3.525 & 217.8 & 0.68 & 2950.7 & 4.0 & 1.4 & $350-483$ \\
1-Propanol & 2.210 & 3.464 & 217.9 & 0.24 & 3189.2 & 3.6 & 1.5 & $293-493$ \\
1-Butanol & 2.808 & 3.426 & 218.1 & 0.11 & 3289.4 & 6.7 & 1.1 & $390-485$ \\
1-Pentanol & 3.228 & 3.398 & 218.9 & 0.04 & 3522.3 & 7.6 & 3.2 & $327-483$ \\
1-Hexanol & 4.002 & 3.401 & 220.2 & 0.01 & 3540.2 & - & 1.5 & $309-409$ \\
\hline
\end{tabular}

\footnotetext{
${ }^{a}$ The parameters are taken from ref. 42 .
} 
For vapor-liquid surface, the initial guess of density profile is as follows:

$$
\rho(z)= \begin{cases}\rho^{1} & z \leqslant 0 \\ \rho^{\mathrm{v}} & z>0\end{cases}
$$

Once the equilibrium profile $\rho_{0}(z)$ and the Helmholtz free energy density $a\left[\rho_{0}(z)\right]$ are determined, the vaporliquid surface tension can be calculated from the following equation:

$$
\gamma=\int_{-\infty}^{\infty}\left[a\left[\rho_{0}(z)\right]-\rho_{0}(z) \mu+p^{\operatorname{coex}}\right] \mathrm{d} z
$$

The calculated vapor-liquid surface tension of methanol and the comparison with experiments is shown in the inset of Figure 1(a).

For vapor-liquid nucleation, solutions to $\delta \Omega[\rho(\boldsymbol{r})] / \delta \rho(\boldsymbol{r})=0$ correspond to saddle points in functional space in systems at a constant temperature and chemical potential. The effective numerical procedures to derive solutions for the Euler-Lagrange equation in open systems have been described in detail by previous authors. ${ }^{25}$ The initial guess of density profile can be expressed as follows:

$$
\rho(r)=\left\{\begin{array}{lc}
\rho^{1} & 0 \leqslant r \leqslant r_{0} \\
\rho^{\mathrm{sv}} & r>r_{0}
\end{array}\right.
$$

where $r_{0}=2 \gamma /(k T \rho \ln S), S=p / p^{\text {coex }}$ stands for supersaturation and $\rho^{\mathrm{sv}}$ is the density of the supersaturated gas surrounding the liquid droplets.

Figure 1 shows the equilibrium density profiles for vapor-liquid surface $(S=1)$ and vapor-liquid nuclei $(S>$ 1) of methanol at given temperature $T=298.15 \mathrm{~K}$. One finds the density profiles broaden with the decrease of supersaturation, which indicates the nuclei have larger size in case of lower pressures. In addition, this figure shows that the densities in the droplet center firstly increase and then decrease with the increase of supersaturation.

When the equilibrium density profile for the critical nucleus, $\rho_{0}(r)$, is obtained, the work of formation $\Delta \Omega$ and the nucleation rate $J$ can be calculated from:

$$
\begin{aligned}
& \Delta \Omega\left[\rho_{0}(\boldsymbol{r})\right]=\Omega\left[\rho_{0}(\boldsymbol{r})\right]-\Omega\left[\rho^{\mathrm{sv}}\right] \\
& J=J_{0} \exp \left[\frac{-\Delta \Omega\left[\rho_{0}(\boldsymbol{r})\right]}{k T}\right]
\end{aligned}
$$

where $J_{0}=\sqrt{2 \gamma /\left(\pi M_{0}\right)} \rho^{\text {sv } 2} / \rho^{1}$ is the rate prefactor and $M_{0}$ is the molecular weight.

Figure 2 shows the nucleation rates of methanol calculated from different approaches (DFT, CNT, KSR model and Scale model), and the comparisons with experimental data. ${ }^{15}$ To make a fair comparison, in the calculations of the mentioned four approaches, the mo- lecular parameters and the intermediate variables are of same values. The molecular parameters are listed in Table 1 . The intermediate variables, e.g., $\rho^{\mathrm{v}}, \rho^{1}, p^{\text {coex }}$ and $\rho^{\mathrm{sv}}$ are predicted by SAFT, and the surface tensions are predicted from Eq. (13).
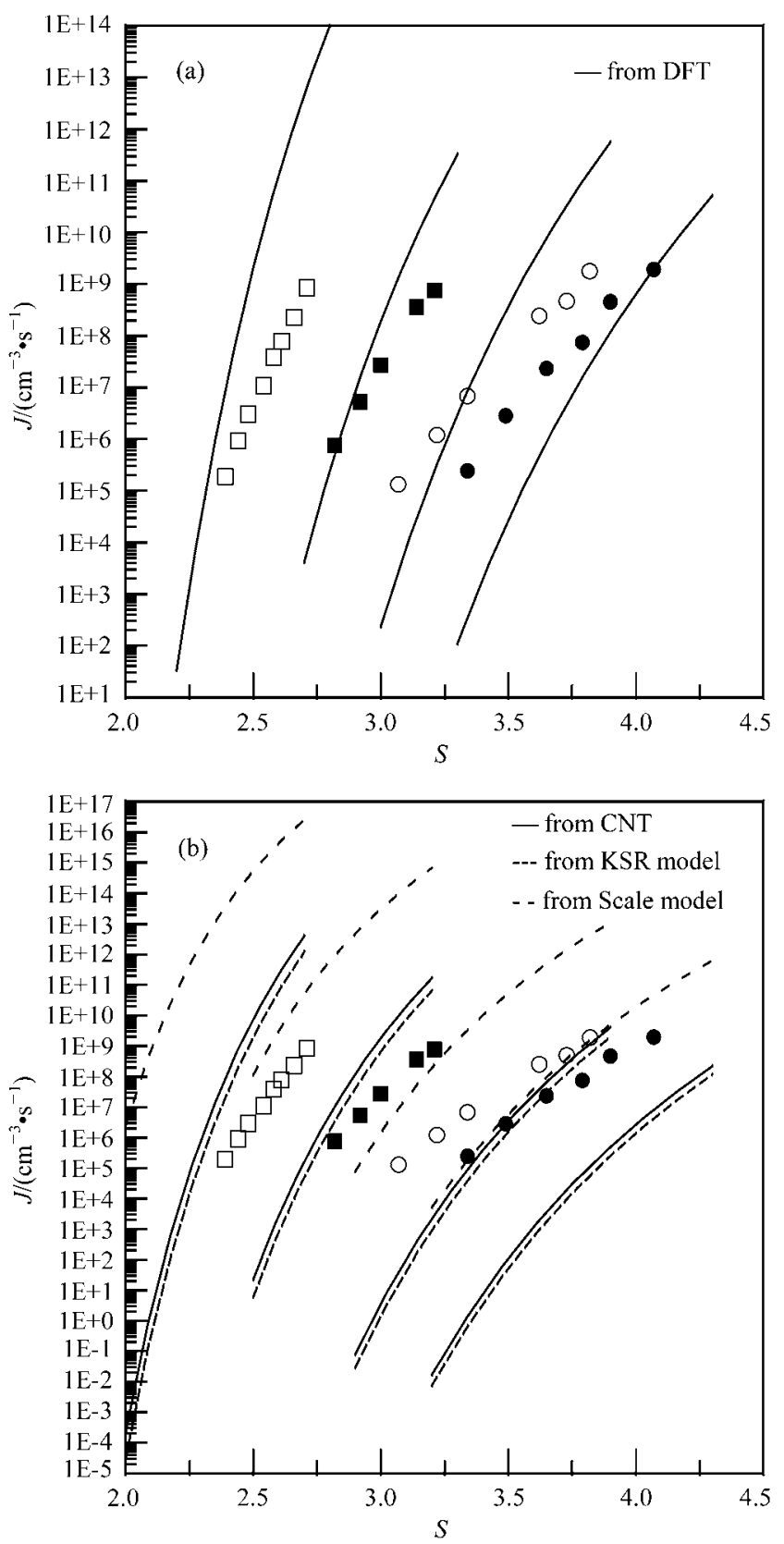

Figure 2 Nucleation rates for methanol. Symbols: experimental data. ${ }^{15}$ : $248 \mathrm{~K}$; O: $258 \mathrm{~K} ; \mathbf{\square}: 278 \mathrm{~K}$; $\square: 298 \mathrm{~K}$.

The nucleation rates predicted by DFT are shown in Figure 2(a). It seems DFT correctly captures the dependence of nucleation rates as a function of supersaturation. From 248 to $298 \mathrm{~K}$, the maximum scales $(\Delta=$ $\left.J_{\mathrm{DFT}} / J_{\text {exp }}\right)$ are respectively $0.0016,75.24,62.38$ and 975.44. The best agreement occurs at the $T=278 \mathrm{~K}$. Taking into account the nucleation rate depends exponentially on the work of formation and any small error in the calculation of $\Delta \Omega(k T)$ will lead to large deviation, 
the DFT calculations for nucleation rates are fairly good. The nucleation rates predicted by CNT, KSR model and Scale model are shown in Figure 2(b). These three approaches also capture the dependence of the nucleation rate as a function of supersaturation, however, the agreements of the predicted values with the experimental data are not satisfactory. The Scale model $(J=$

$J_{0} \exp \left[-\frac{16 \pi \varpi}{3}\left(\frac{T_{\mathrm{c}}}{T}-1\right)^{3} /(\ln S)^{2}\right], J_{0}=10^{26} \mathrm{~cm}^{-3} \cdot \mathrm{s}^{-1}$,

$\varpi=1.5$ and $T_{\mathrm{c}}=512.7 \mathrm{~K}$ ) yields reasonable nucleation rate at relatively low temperature. However, with the increase of temperature, it significantly overestimates the nucleation rates. The KSR model $\left(\Delta \Omega_{\mathrm{KSR}}=16 \pi \gamma^{3} /\right.$ $\left\{3 \rho^{1} k T \ln \left[p_{1} / p_{1}^{\text {coex }}\right]\right\}^{2}, p_{1}$ and $p_{1}^{\text {coex }}$ respectively stand for the partial pressure of monomers in supersaturated gas and in equilibrium bulk gas) and $\operatorname{CNT}\left(\Delta \Omega_{\mathrm{KSR}}=\right.$ $16 \pi \gamma^{3} /\left[3 \rho^{1} k T \ln S\right]^{2}$ ) reasonably predict the nucleation rates at relatively high temperatures, however, at low temperatures, the nucleation rates of methanol are seriously underestimated. It is worth noting that besides DFT, DGT can also be used to investigate the microstructure of nucleus, including density profile and number of particles within the nucleus. Fu and Liu ${ }^{42}$ applied DGT to the nucleation properties of $n$-alcohols, and the agreement between calculated and experimental data in their work is better than that in our work. DFT is theoretically better than DGT because in the calculations of interfacial properties and nucleation properties, DFT only needs the molecular parameters regressed from bulk properties as input. However, in the calculation of DGT, for the determination of both surface tension ${ }^{53-55}$ and nucleation rates, ${ }^{42}$ additional influence parameters are need.

Figure 3 shows the predicted nucleation rates of ethanol and the comparisons with experimental data. ${ }^{15}$ Similar to the information presented in Figure 2(a), the predictions from DFT are fairly good. From 258 to 318 $\mathrm{K}$, the maximum scales are respectively $0.0079,0.0027$, $0.0024,0.051,75.2,996.4$ and 3492.5. The best agreement occurs at the $T=288 \mathrm{~K}$. Figure $3(\mathrm{~b})$ shows that both KSR model and CNT significantly underestimate the nucleation rates at relatively low temperatures. In addition, these two approaches give very close predictions for ethanol because compared with the critical temperature, the investigated temperatures (from 258 to $318 \mathrm{~K}$ ) are relatively low, and at low temperatures, the calculated molar fraction of monomers in equilibrium bulk gas $\left(x_{1}^{\mathrm{e}}\right)$ is very close to that in supersaturated gas $\left(x_{1}^{\mathrm{s}}\right)$, hence $p_{1} / p_{1}^{\text {coex }}$ is very close to $p / p^{\text {coex }}$. The Scale model significantly overestimates the nucleation rates from 258 to $318 \mathrm{~K}$. The agreement can be improved if one adjusts the values of $J_{0}$ and $\varpi$, however, the improvement is slight and the predictions are still unsatisfactory compared with DFT.

The predictions of nucleation rates from DFT and CNT, as well as the comparisons with experimental
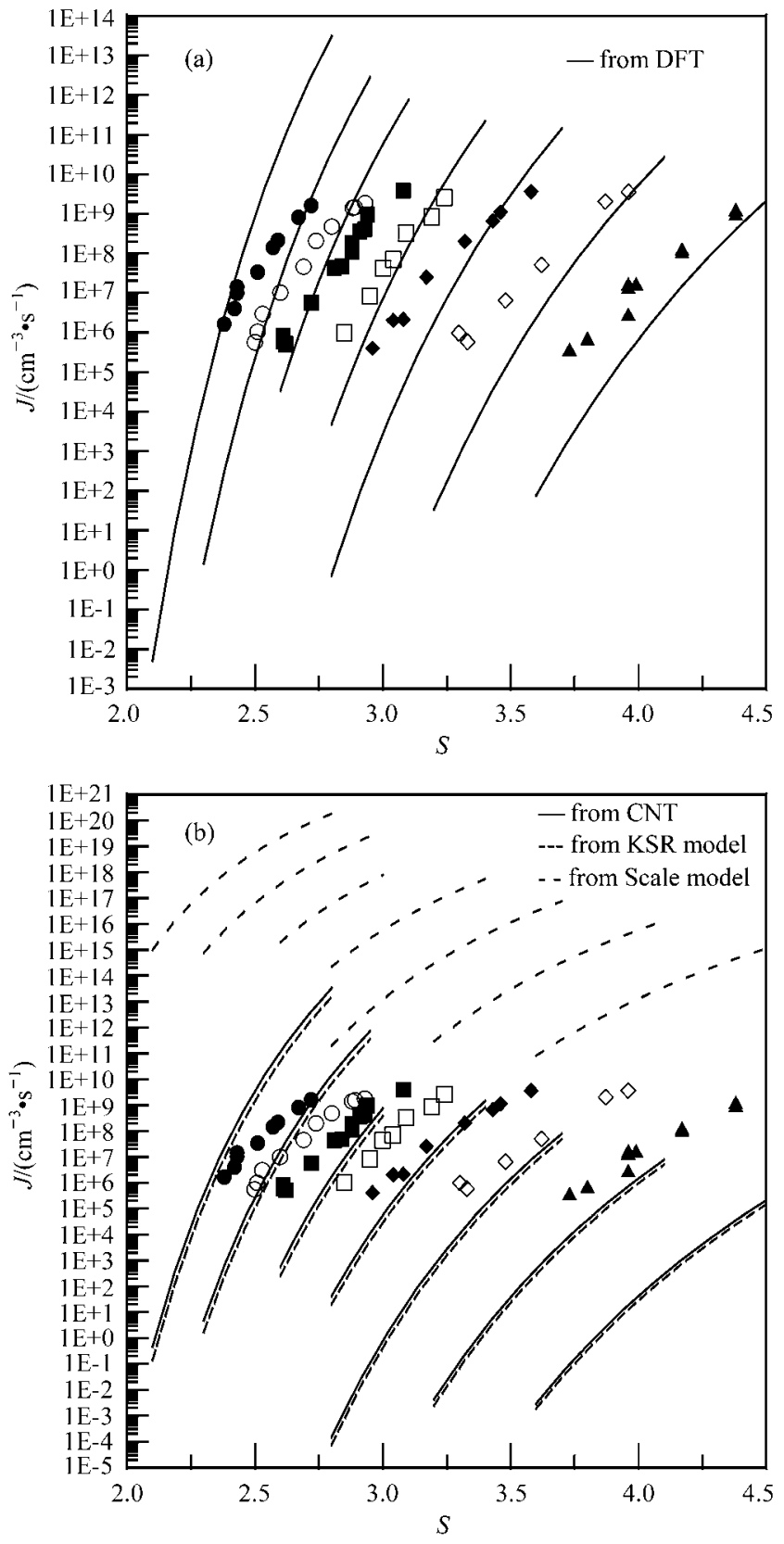

Figure 3 Nucleation rates for ethanol. Symbols: experimental data. $^{15} \boldsymbol{\Delta}: 258 \mathrm{~K} ; \diamond: 268 \mathrm{~K} ; \diamond: 278 \mathrm{~K} ; \square: 288 \mathrm{~K} ; \mathbf{\square}: 298 \mathrm{~K}$; ○: $308 \mathrm{~K} ; 0: 318 \mathrm{~K}$.

data, ${ }^{15}$ are respectively shown in Figures $4-7$ for 1-propanol, 1-butanol, 1-pentanol and 1-hexanol. The predictions from KSR model and Scale model are not presented in these figures because the results from KSR model are almost the same as those from CNT, and $J_{0}=$ $10^{26} \mathrm{~cm}^{-3} \cdot \mathrm{s}^{-1}$ and $\varpi=1.5$ in Scale model are not universal for $n$-alcohol series.

DFT satisfactorily predicts the nucleation rates for 1-propanol at 258, 278 and $298 \mathrm{~K}$ with the maximum scales respectively $0.021,0.046$ and 3.88 , but seriously overestimates those at $318 \mathrm{~K}$, with the maximum scale 1940.2. Contrary to the predictions from DFT, CNT seriously underestimates the nucleation rates at 258, 278 and $298 \mathrm{~K}$, yet accurately predicts those at $318 \mathrm{~K}$, as 


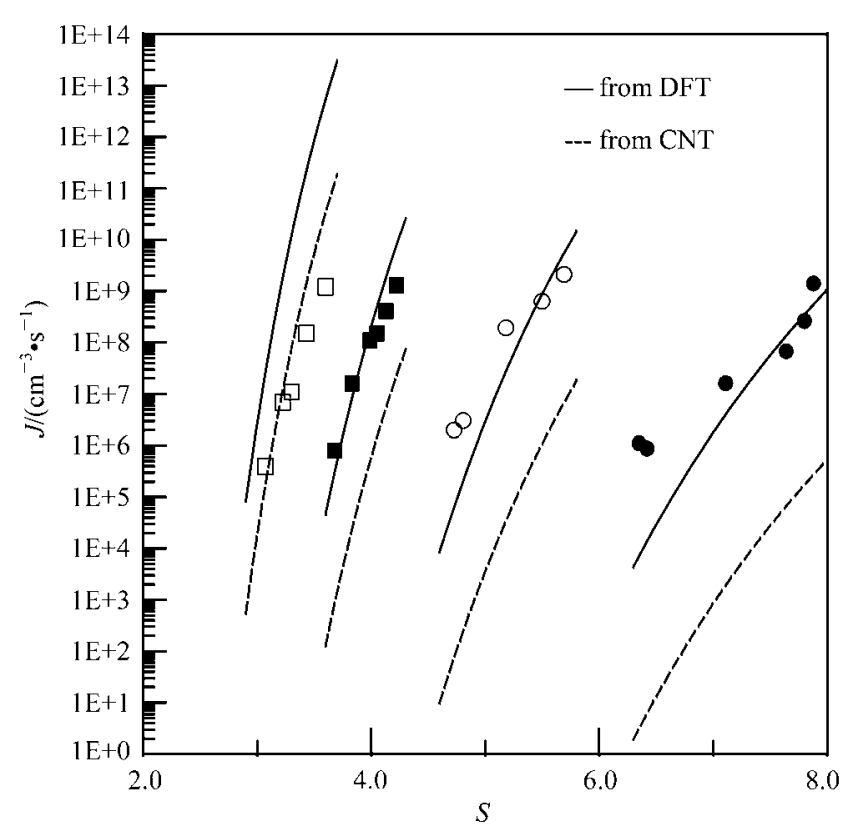

Figure 4 Nucleation rates for $n$-propanol. Symbols: experimental data. ${ }^{15}$ : $258 \mathrm{~K}$; О: $278 \mathrm{~K}$; $\mathbf{\square}: 298 \mathrm{~K} ; \square: 318 \mathrm{~K}$.

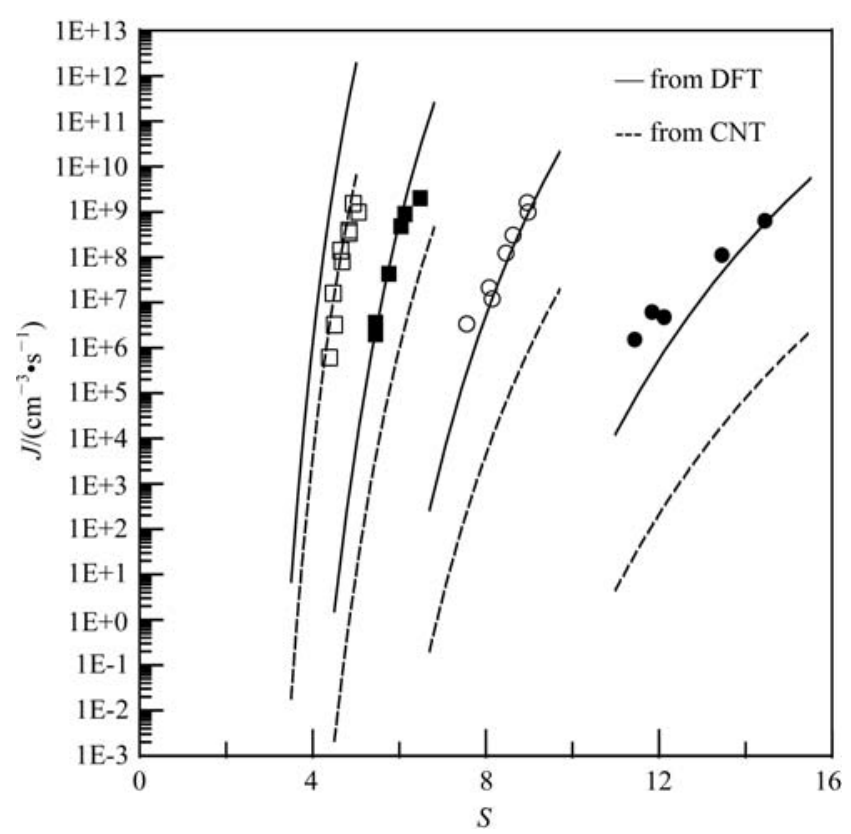

Figure 5 Nucleation rates for $n$-butanol. Symbols: experimental data. ${ }^{15}$ : $258 \mathrm{~K}$; O: $278 \mathrm{~K} ; \mathbf{\square}: 298 \mathrm{~K} ; \square: 318 \mathrm{~K}$.

shown in Figure 4. The predictions for 1-butanol, as shown in Figure 5, are very similar to those for 1-propanol. The maximum scales at 258, 278, 298 and $318 \mathrm{~K}$ are respectively $0.045,0.12,7.13$ and 248.79 . For 1-pentanol, DFT satisfactorily predicts the nucleation rates at $278 \mathrm{~K}$ with the maximum scale 0.333 but slightly overestimates those at $298 \mathrm{~K}$ with the maximum scale 297.4, however, CNT underestimates the nucleation rates at both temperatures, as shown in Figure 6. For 1-hexanol, although the molecular parameters are regressed by fitting to the saturation liquid densities only, the DFT predicts the nucleation rates fairly good at $288,298,308,318$ and $328 \mathrm{~K}$, with the maximum

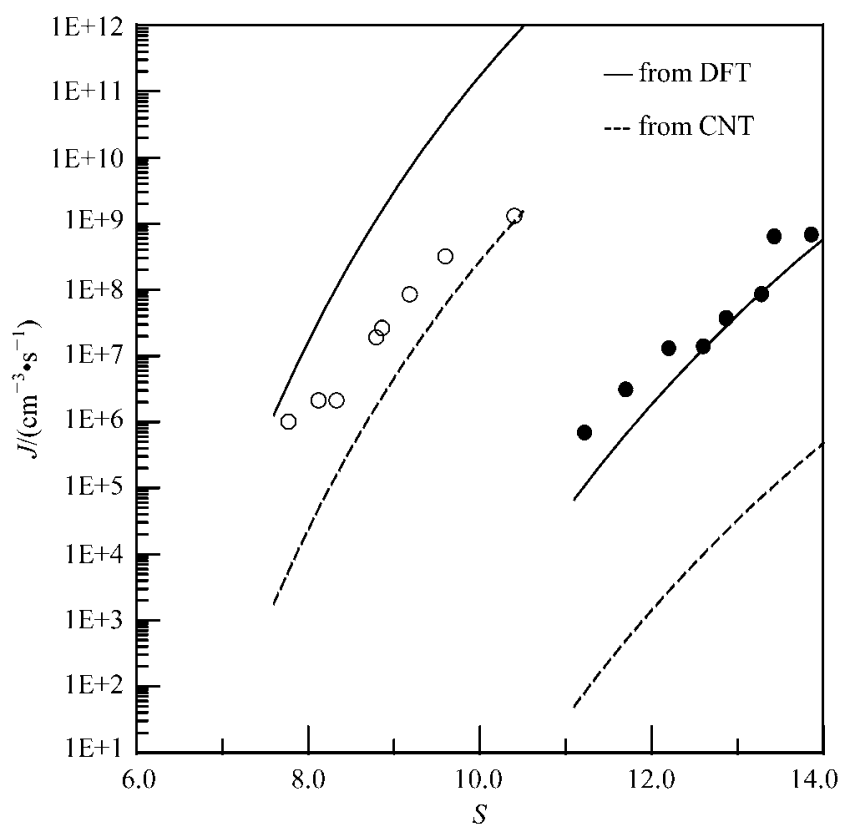

Figure 6 Nucleation rates for $n$-pentanol. Symbols: experimental data. ${ }^{15}$ : $278 \mathrm{~K}$; O: $298 \mathrm{~K}$.

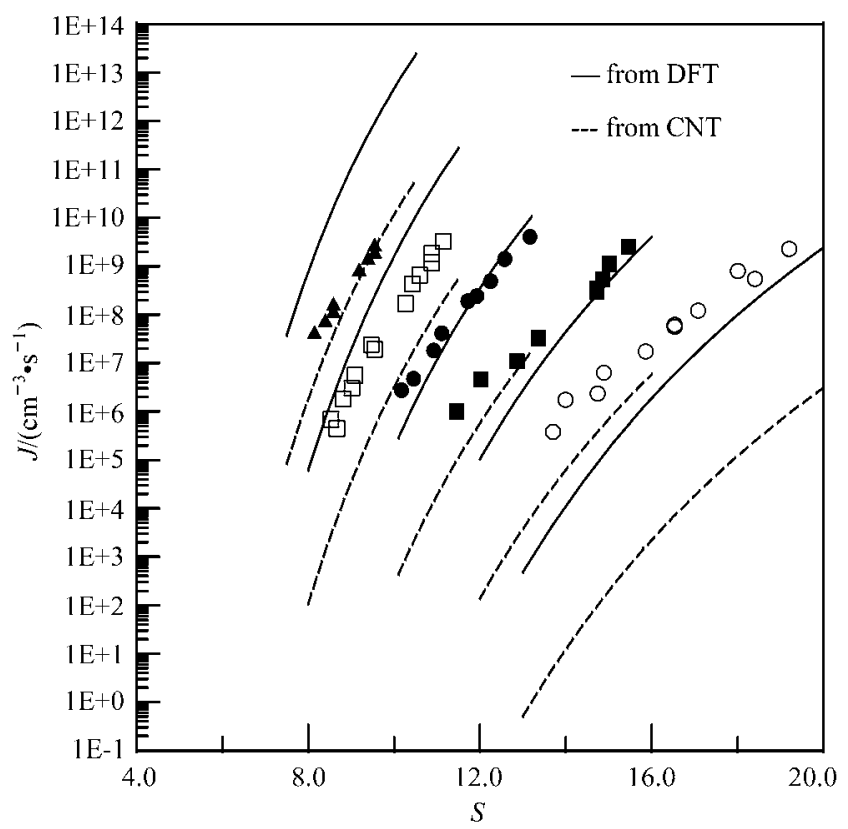

Figure 7 Nucleation rates for $n$-hexanol. Symbols: experimental data. ${ }^{15}$ O: $288 \mathrm{~K} ; \mathbf{\square}: 298 \mathrm{~K}$; $: 308 \mathrm{~K} ; \square: 318 \mathrm{~K} ; \boldsymbol{\Delta}: 328 \mathrm{~K}$.

scales $0.013,0.051,0.25,31.4$ and 336.3 , respectively. However, CNT only satisfactorily predicts the nucleation rates at $328 \mathrm{~K}$, at other four temperatures, the nucleation rates are seriously underestimated.

\section{Conclusion}

In summary, the SAFT and WCA approximation are combined to establish an EOS applicable for the bulk and nucleation properties for $n$-alcohols. The molecular parameters for $6 n$-alcohols are determined by fitting to the equilibrium bulk properties and then used to predict the nucleation rates within the framework of DFT. Re- 
sults show that the combination of DFT and SAFT is a successful approach for vapor-liquid nucleation of $n$-alcohols. Firstly, by formulating the intermolecular interactions via Lennard-Jones potential in a WCA approximation, SAFT is able to accurately describe the bulk properties and convenient to be extended to vapor-liquid surface, which are very important for the calculations of interfacial and nucleation properties; secondly, among the various approaches applicable for the nucleation rates of $n$-alcohols, DFT is the most accurate one. For the investigated $6 n$-alcohols, the maximum scales $J_{\mathrm{DFT}} / J_{\text {exp }}$ predicted by DFT are around 1 to 3 orders of magnitude. Taking into account that the molecular parameters regressed from bulk properties are the unique input, the prediction is fairly good.

\section{References}

1 Gibbs, J. W. The Scientific Papers of J. W. Gibbs, Dover, New York, 1961.

2 Volmer, M.; Weber, A. Z. Phys. Chem. 1926, 119, 277.

3 Farkas, A. Z. Phys. Chem. 1927, 125, 236.

4 Becker, R.; Doring, W. Ann. Phys. 1935, 24, 719.

5 Frenkel, J. J. Chem. Phys. 1939, 7, 538.

6 Zeldovich, J. B. Acta Physico-chimica U. S. S. R. 1943, 18, 1.

7 Frenkel, J. Kinetic Theory of Liquids, Dover, New York, 1955.

8 Abraham, F. F. Homogeneous Nucleation Theory, Academic, New York, 1974.

9 Kashchiev, D. Nucleation: Basic Theory with Applications, Butterworth-Heinemann, Oxford, 2000.

10 Debenedetti, P. G. Metastable Liquids: Concepts and Principles, Princeton University Press, Princeton NJ, 1996.

11 Schmitt, J. L.; Whitten, J.; Adams, G. W.; Zalabsky, R. A. J. Chem. Phys. 1990, 92, 3693.

12 Hung, C. H.; Krasnopoler, M.; Katz, J. L. J. Chem. Phys. 1989, 90, 1856.

13 Wolk, J.; Strey, R. J. Phys. Chem. B 2001, 105, 11683.

14 Agarwal, G.; Heist, R. H. J. Chem. Phys. 1980, 73, 902.

15 Strey, R.; Wagner, P. E.; Schmeling, T. J. Chem. Phys. 1986, 84, 2325.

16 Hale, B. N. Phys. Rev. A 1986, 33, 4156.

17 Hale, B. N. Metallurgical Trans. A 1992, 23, 1986.

18 Katz, J. L.; Saltsburg, H.; Reiss, H. J. Colloid Interface Sci. 1966, $21,560$.

19 Talanquer, V.; Oxtoby, D. W. J. Chem. Phys. 2000, 112, 851.

20 Evans, R. Adv. Phys. 1979, 28, 143.

21 Evans, R. In Fundamentals of Inhomogeneous Fluids, Ed.: Henderson, D., Dekker, New York, 1992.
22 Abraham, F. F. Phys. Rep. 1979, 53, 93.

23 Cahn, J. W.; Hilliard, J. E. J. Chem. Phys. 1958, 28, 258.

24 Cahn, J. W.; Hilliard, J. E. J. Chem. Phys. 1959, 31, 688.

25 Oxtoby, D. W.; Evans, R. J. Chem. Phys. 1988, 89, 7521.

26 Harrowell, P. R.; Oxtoby, D. W. J. Chem. Phys. 1984, 80, 1639.

27 Zeng, X. C.; Oxtoby, D. W. J. Chem. Phys. 1991, 94, 4472.

28 Zeng, X. C.; Oxtoby, D. W. J. Chem. Phys. 1991, 95, 5940.

29 Oxtoby, D. W.; Harrowell, P. R. J. Chem. Phys. 1992, 96, 3834.

30 Oxtoby, D. W.; Kashchiev, D. J. Chem. Phys. 1994, 100, 7665.

31 Fu, D. Chin. Phys. Lett. 2005, 22, 1378.

32 Fu, D.; Liao, T. Chin. Phys. 2007, 16, 3475.

33 Fu, D.; Liao, T. Chin. Phys. Lett. 2007, 24, 2804.

34 Shen, Y. C.; Oxtoby, D. W. J. Chem. Phys. 1996, 105, 6517.

35 Shen, Y. C.; Oxtoby, D. W. Phys. Rev. Lett. 1996, 77, 3585.

36 Talanquer, V.; Oxtoby, D. W. J. Chem. Phys. 1998, 109, 223.

37 Talanquer, V.; Cunningham, C.; Oxtoby, D. W. J. Chem. Phys. 2001, 114, 6759.

38 Christopher, P. S.; Oxtoby, D. W. J. Chem. Phys. 2003, 119, 10330.

39 Napari, I.; Laaksonen, A.; Talanquer, V.; Oxtoby, D. W. J. Chem. Phys. 1999, 110, 5906.

40 Talanquer, V. J. Phys. Chem. B 2007, 111, 3438.

41 Fu, D.; Li, X.-S. J. Phys. Chem. C 2007, 111, 13938.

42 Fu, D.; Liu, J. M. Chin. J. Chem. 2009, 27, 235.

43 Andersen, H. C.; Weeks, J. D.; Chandler, D. Phys. Rev. A 1971, 4, 1597.

44 Chapman, W. G.; Gubbins, K. E.; Jackson, G.; Radosz, M. Fluid Phase Equilib. 1989, 52, 31.

45 Huang, S. H.; Radosz, M. Ind. Eng. Chem. Res. 1990, 29, 2284.

46 Wertheim, M. S. J. Stat. Phys. 1986, 42, 459.

47 Wertheim, M. S. J. Stat. Phys. 1986, 42, 477.

48 Carnahan, N. F.; Starling, K. E. J. Chem. Phys. 1969, 51, 635.

49 Barker, J. A.; Henderson, D. J. Chem. Phys. 1967, 47, 4714.

50 Verlet, L.; Weis, J. J. Phys. Rev. A 1972, 5, 939.

51 Beaton, C. F.; Hewitt, G. F. Physical Property Data for the Design Engineer, Hemisphere Publishing Corporation, New York, 1989.

52 Smith, B. D.; Srivastava, R. Thermodynamic Data for Pure Components, Elsevier, Amsterdam, 1986.

53 Fu, D. Ind. Eng. Chem. Res. 2007, 46, 7378.

54 Fu, D. J. Phys. Chem. B 2006, 110, 19575.

55 Fu, D. Chin. J. Chem. 2006, 24, 1315.

(E1003032 Pan, B.) 